\title{
Aplicação da escala Spinal Alignment and Range of Motion Measure (SAROMM) em crianças e adultos com paralisia cerebral, em uma instituição de abrigagem de Porto Alegre (RS)
}

Application of the scale Spinal Alignment and Range of Motion Measure (SAROMM) in children and adults with cerebral palsy in a compartment institution in Porto Alegre (RS) Aplicación de la escala Spinal Alignment and Range of Motion Measure (SAROMM) en niños y adultos con parálisis cerebral de una institución de refugio en Porto Alegre (RS)

Emanuele Priscila Alves Cominetti', Laís Rodrigues Gerzson², Carla Skilhan de Almeida ${ }^{3}$

RESUMO | O objetivo do estudo foi descrever o perfil de crianças e adultos institucionalizados com Paralisia Cerebral, bem como, suas alterações musculoesqueléticas, alinhamento da coluna vertebral e amplitude de movimento; também, traçar estratégias para minimizar o avanço das deformidades já instaladas. Estudo de caráter transversal e descritivo. Crianças e adultos com Paralisia Cerebral de uma Instituição de abrigagem da cidade de Porto Alegre/RS foram avaliados $(n=28)$. Utilizou-se a escala Spinal Alignment and Range of Motion Measure (SAROMM) para avaliar as deformidades e Gross Motor Function Classification System (GMFCS) para classificar o nível funcional. $96 \%$ dos sujeitos foram do tipo espástico; 42,85\% apresentaram nível motor $\vee$ do total dos participantes; as maiores deformidades encontradas foram em joelho, quadril e coluna avaliados pela escala de SAROMM, associado com a idade mais avançada e quadro de quadriplegia espástica, com diagnóstico clínico em prontuário. Sujeitos maiores de 20 anos obtiveram uma pontuação média de 68,7 (varia de 0 a 104) e sujeitos menores de 20 anos, sua pontuação média foi de 55,1 pontos, sendo quanto menor, melhor o alinhamento e menos deformidades. Sujeitos com PC de um local de abrigagem apresentaram, na sua maioria, espasticidade do tipo bilateral dos quatro membros, nível de GMFCS predominante foi o $\mathrm{V}$ e alterações articulares em joelhos, quadril e coluna vertebral como maiores deformidades encontrada. As estratégias devem ser traçadas e iniciadas - mais cedo possível para diminuir e ou minimizar deformidades e a escala SAROMM pode ser uma escolha para avaliar esse público.

Descritores | Paralisia Cerebral; Avaliação da Deficiência; Fisioterapia.

\begin{abstract}
This study described the profile of institutionalized children and adults with cerebral palsy (CP), as well as their musculoskeletal alterations, spinal alignment and range of motion; and to outline strategies to minimize the advance of already present deformities. This is a cross-sectional and descriptive study. Children and adults with cerebral palsy from a shelter in the city of Porto Alegre, Rio Grande do Sul were evaluated ( $\mathrm{n}=28$ ). The Spinal Alignment and Range of Motion Measure (SAROMM) scale was used to assess deformities and the Gross Motor Function Classification System (GMFCS) was used to classify the functional level. 96\% of the subjects were spastic; $42.85 \%$ of the total number of participants had motor level V; the greatest deformities found were in the knee, hip and spine, evaluated by the SAROMM Scale, associated with older age and spastic quadriplegia, with clinical diagnosis in the medical record. Subjects over 20 years old had an average
\end{abstract}

Estudo realizado na Casa do Menino Jesus de Praga, Porto Alegre (RS).

Universidade Federal do Rio Grande do Sul (UFRGS) - Porto Alegre (RS), Brasil. E-mail: manu_ac14@hotmail.com. Orcid: 0000-0003-3213-6810

2Universidade Federal do Rio Grande do Sul (UFRGS) - Porto Alegre (RS), Brasil. E-mail: gerzson.lais@yahoo.com.br. Orcid: 0000-0002-0911-9820

3Universidade Federal do Rio Grande do Sul (UFRGS) - Porto Alegre (RS), Brasil. E-mail: carlaskilhan@gmail.com. Orcid: 0000-0003-1271-2876 
score of 68.7 (ranges from 0 to 104) and subjects under 20 years old, their average score was 55.1 points, wherein the lower the value the better the alignment and the less deformities. Subjects with CP of a shelter location had, mostly, bilateral spasticity of the four limbs, their predominant GMFCS level was $V$ and the major deformities found were joint changes in knees, hip and spine. Strategies should be drawn and executed as early as possible to reduce deformities and the SAROMM scale may be a choice to evaluate this audience. Keywords | Cerebral Palsy; Disability Evaluation; Physiotherapy.

RESUMEN | El objetivo de este estudio fue describir el perfil de niños y adultos institucionalizados con parálisis cerebral (PC), sus cambios musculoesqueléticos, alineación espinal y rango de movimiento, así como elaborar estrategias que mitigan el avance de las deformidades ya instaladas. Se trata de un estudio transversal y descriptivo. Se evaluaron a niños y adultos con parálisis cerebral en una institución de refugio en la ciudad de Porto Alegre, ubicada en el estado de Rio Grande do Sul (n=28). Se utilizó la escala Spinal Alignment and Range of Motion Measure (SAROMM) para evaluar las deformidades y el
Sistema de Clasificación de la Función Motora Gruesa (GMFCS) para clasificar el nivel funcional. El 96\% de los sujetos eran espásticos; el 42,85\% tenían nivel motriz V; las mayores deformidades encontradas fueron en las rodillas, la cadera y la columna evaluadas por la escala SAROMM, que se asociaban a mayor edad y la cuadriplejía espástica con diagnóstico en la historia clínica. Los sujetos mayores de 20 años tuvieron un puntaje promedio de 68,7 (que oscila de 0 a 104), y los sujetos menores de 20 años presentaron un puntaje promedio de 55,1 puntos, por tanto, cuanto menor la edad, mejor es la alineación y menos deformidades. Los sujetos con PC de un local de refugio presentaron, en su mayoría, espasticidad de tipo bilateral en las cuatro extremidades, predominancia del nivel $\mathrm{V}$ de GMFCS y cambios articulares en rodillas, cadera y columna como las mayores deformidades encontradas. Se debe elaborar y comenzar estrategias lo antes posible para disminuir o mitigar las deformidades de esta población, y la escala SAROMM puede ser una opción para evaluarla.

Palabras clave | Parálisis Cerebral; Evaluación de la Discapacidad; Fisioterapia.

\section{INTRODUÇÃO}

O sujeito morador de uma instituição de abrigagem, além de experimentar o sentimento de abandono, desamor ou desconsideração, experimenta a privação do convívio familiar, às vezes impossível de ser contornado. Mais ainda, um sujeito morador de uma instituição de abrigagem com Paralisia Cerebral (PC), funcionalmente dependente de seus cuidadores, são praticamente esquecidos ${ }^{1}$. Por muito tempo, esses sujeitos permaneceram (ou ainda permanecem) sem nenhum tipo de intervenção (motora, cognitiva, aprendizagem, linguagem, espiritual etc.). Apenas intervenção de higiene e alimentação. É necessário que esta população seja vista, estudada, que a comunidade científica traga informações novas e significativas destes sujeitos, pois parece ser escassos os estudos nesta área ${ }^{1}$.

A PC é uma enfermidade não progressiva, causada por uma lesão no cérebro imaturo, representando a mais comum desordem neuromuscular infantil. A lesão pode ocorrer durante o período pré-natal, perinatal ou pós-natal e as causas podem ser: congênitas, genéticas, inflamatórias, infecciosas, anóxicas, traumáticas e metabólicas ${ }^{2,3}$. Pode ser classificada de acordo com a região cerebral acometida, conforme a severidade do comprometimento ou pela distribuição topográfica da lesão. Quanto ao acometimento cerebral, a PC pode ser subdividida em espástica, atáxica, discinética, distônica, mista e hipotônica. A distribuição topográfica se classifica como bilateral (os dois lados do corpo acometido) ou unilateral (um hemicorpo acometido) ${ }^{4}$.

A PC tem uma crescente prevalência, ou seja, cerca de 1,5 a 4 crianças a cada 1000 nascidas vivas tem PC, gerando alto custo econômico e impactando negativamente na qualidade de vida ${ }^{5}$. Os danos provocados no sistema nervoso imaturo, associados à assistência terapêutica tardia acarretam um prognóstico ruim, relacionado ao desenvolvimento neuropsicomotor de crianças com $\mathrm{PC}^{6}$. Apesar da lesão cerebral permanecer estática em seu tamanho, as alterações musculoesqueléticas resultantes irão invariavelmente progredir em decorrência da espasticidade, da fraqueza muscular e da deficiência do crescimento longitudinal do esqueleto ${ }^{7}$.

Posturas e padrões de movimentos atípicos podem desencadear atraso na aquisição das habilidades motoras. Pessoas com PC são mais propensas a ter uma diminuição da amplitude de movimento (ADM) devido à mobilidade reduzida e à presença de espasticidade e/ou distonia. A perda da amplitude articular é uma das principais preocupações no manejo em longo prazo de crianças com PC. Deformidades como escoliose, obliquidade pélvica e luxação de quadril são mais frequentes em adultos gravemente acometidos, geralmente aqueles com PC do 
tipo espástica bilateral ou aqueles que não deambulam ${ }^{8,9}$. As contraturas musculares, deformidade nos pés e osteoartroses, por outro lado, podem ser encontradas em todos os tipos de $\mathrm{PC}^{10}$. Devido ao impacto negativo das alterações musculoesqueléticas sobre a qualidade de vida dos pacientes com PC, torna-se de extrema importância avaliar este paciente, principalmente quando não existe uma família que possa favorecer melhores condições de vida.

A sequela de espasticidade é a mais frequente encontrada nos casos de PC, acometendo mais de 70\% dos $\operatorname{casos}^{11}$. A espasticidade pode acarretar alterações musculoesqueléticas secundárias à alteração do tônus muscular. Dentre as alterações musculoesqueléticas, aquelas que acometem os membros inferiores são mais frequentes com rotação medial de quadril, flexão de joelhos, pés equinovaros, corroborando com os nossos achados. Estas alterações podem ser decorrentes do encurtamento adaptativo dos tecidos moles, perda da ADM e alterações biomecânicas da congruência articular, uma vez que alguns músculos dos membros inferiores agem em mais de uma articulação ${ }^{12}$. A espasticidade ocorre devido à lesão hipóxico-isquêmica e sangramentos, principalmente da artéria cerebral média (ACM) nas áreas corticais e subcorticais, classicamente consideradas como sistema motor primário, como giros pré-central, cápsula interna e substância branca ${ }^{13-15}$.

Para isso, existem escalas de avaliação que são ferramentas que coletam dados sobre o paciente e se relacionam com os objetivos de tratamento traçados, a fim de realizar uma avaliação pré e pós-intervenção, criar indicadores de saúde, ou discriminar o perfil do paciente tratado. São utilizados para medir, descrever, prever ou avaliar mudanças ao longo do tempo, e também para estabelecer metas funcionais e planejamento de intervenções. O estudo realizado por Bartlett e Purdie ${ }^{16}$, descreveu o desenvolvimento e testes preliminares da escala Spinal Alignment and Range of Motion Measure (SAROMM). Esse estudo demonstrou que SAROMM tem confiabilidade e validade suficientes para uso em crianças com PC em ambientes clínicos e de pesquisa por profissionais da reabilitação.

O que originou este estudo foi a ideia de se verificar o perfil de uma população (de diversas faixas etárias com PC) de abrigagem, que não recebia no passado um tratamento precoce multidisciplinar, onde esses sujeitos apresentaram contraturas e deformidades adquiridas. A proposta era conhecer para programar estratégias de tratamento baseados em evidências. Porém, chamou atenção que são escassos os artigos que descrevem esta população, sendo extremamente necessária as pesquisas neste contexto. Então é importante estudá-las com profundidade. Esses dados poderiam predizer algo concreto para as populações seguintes que realizam uma reabilitação multidisciplinar precoce. Será que futuramente poderemos mostrar a importância do início precoce da reabilitação, comparando com aqueles que não tiveram a mesma oportunidade?

Assim, este estudo teve como objetivo descrever o perfil de crianças e adultos institucionalizados com PC (esta população nunca foi estudada) e as alterações musculoesqueléticas, alinhamento da coluna vertebral e ADM dos mesmos, bem como, traçar estratégias para minimizar o avanço das deformidades já instaladas e utilizar uma nova forma de avaliação pré e pós intervenção terapêutica.

\section{METODOLOGIA}

\section{Delineamento e participantes}

Este se caracteriza como um estudo de caráter transversal e descritivo, com amostra não probabilística por conveniência, de modo que participaram da pesquisa todos os sujeitos moradores da instituição. A coleta de dados foi realizada entre os meses de agosto a dezembro de 2017, sendo a amostra constituída pelas crianças e adultos com PC, moradores de uma Instituição de Abrigagem localizada na cidade de Porto Alegre/RS. Atualmente, a instituição abriga 35 crianças e adultos com lesão neurológica e deficiências múltiplas, com idades entre quatro a 40 anos. $\mathrm{O}$ convite para a participação da pesquisa foi feito para os responsáveis legais dos sujeitos, visto que a guarda dos abrigados era da instituição e os acolhidos possuíam déficit intelectual e não verbalizavam.

Os critérios de inclusão estabelecidos foram: (a) ser morador do abrigo por mais de um ano; (b) ter diagnóstico de PC; (c) não ter impedimentos físicos e psíquicos para realizar os testes. Foram excluídos da pesquisa: não possuírem diagnóstico de $\mathrm{PC}$; ou (b) não tolerarem o toque e manuseios, ou (c) por apresentarem cirurgia na coluna vertebral (artrodese), e, ainda, que (d) apresentassem dificuldades de posicionamento para a realização do teste.

\section{Instrumentos e procedimentos de coleta}

Inicialmente, foi solicitada a autorização para a realização do estudo na instituição e a assinatura do 
Termo de Consentimento Livre e Esclarecido (TCLE). Após a autorização, foram marcadas as datas para as avaliações dos abrigados.

As avaliações foram conduzidas em uma sala do setor, específica para esse fim, com mobília adequada. A equipe para a coleta dos dados foi composta por dois profissionais da fisioterapia. Houve um treinamento prévio de duas semanas para padronizar a avaliação.

Foi utilizado um goniômetro para avaliação da ADM, uma maca para posicionar os pacientes em decúbito dorsal e um banco para realizar a avaliação do paciente na postura sentada. Além disso, foi utilizada a escala Spinal Alignment and Range of Motion Measure (SAROMM) que consta de 26 itens. Contém quatro itens para o alinhamento da coluna vertebral e 11 itens para a amplitude de movimento, testados bilateralmente. Cada item é pontuado em uma escala de 0 a 4 pontos: 0 - significa que não há limitações de alinhamento e a correção é ativa; 1 - significa bom alinhamento com correção passiva; 2 - é atribuído quando a limitação se reduz quase totalmente na correção passiva e há deformidade mínima; 3 - significa que a limitação quase não é redutível de forma passiva e há uma deformidade moderada; 4 - é atribuído quando a limitação não é redutível e a deformidade é grave ${ }^{16}$.

O alinhamento da coluna vertebral foi determinado pela soma das pontuações para os quatro primeiros itens (pontuação de 0 a 16). A amplitude de movimento e a extensibilidade foram determinadas pela soma dos valores dos itens de 5 a 26 (pontuação de 0 a 88). A pontuação total da SAROMM (alinhamento da coluna vertebral de 0 a 16 e amplitude de movimento e a extensibilidade de 0 a 88) pode variar de 0 a 104. Os valores mais baixos indicam um desvio mínimo do alinhamento da coluna vertebral e ADM, enquanto contagens elevadas indicam desvios e limitações significantes. A escala fornece um método confiável para estimar os desvios globais de alinhamento e limitações da amplitude de movimento da coluna vertebral ${ }^{16}$.

Os pacientes também foram classificados conforme Gross Motor Function Classification System (GMFCS). Esta classificação tem importante aplicabilidade clínica nesta população e baseia-se na gravidade do comprometimento funcional, assim como para estabelecer o desenvolvimento esperado em longo prazo. É baseado no movimento iniciado voluntariamente, com ênfase no sentar, transferências e mobilidade. Os pacientes são distribuídos em cinco níveis de gravidade, conforme grau de limitação da função motora grossa, a saber: Nível I - Anda sem restrições (domicílio e comunidade). Velocidade, coordenação e equilíbrio prejudicados; Nível II - Anda com restrições no domicílio e comunidade mesmo em superfícies planas, anda de gato em casa e apresenta dificuldades para pular e correr; Nível III - Anda utilizando um dispositivo manual de mobilidade como andadores e muletas, sobe escadas segurando em corrimão e depende da função dos membros superiores para tocar a cadeira de rodas para longas distâncias; Nível IV - Automobilidade com limitações; pode utilizar mobilidade motorizada. Faz transferências com a ajuda de um adulto, anda com andador para curtas distâncias com dificuldades em superfícies irregulares; Nível V - Transportado em uma cadeira de rodas manual, necessita de adaptações para sentar-se, totalmente dependente em atividades de vida diária e em locomoção e pode tocar cadeira de rodas motorizada com adaptações ${ }^{17}$.

\section{Análise estatística}

Os dados coletados de todas as avaliações foram armazenados em um banco de dados do Software Statistical Package for the Social Sciences (SPSS), versão 22.0. A análise estatística descritiva foi realizada por meio do cálculo de porcentagem, média.

\section{RESULTADOS}

A amostra totalizou 28 participantes, 15 do sexo masculino e 13 do feminino, com idades entre seis a 37 anos, totalizando uma média de 21,7 anos. Essa grande variabilidade de idade é uma característica da maioria das instituições que abrigam PC. Doze participantes apresentaram GMFCS nível V (42,8\%), dez apresentaram nível IV (35,7\%), quatro apresentaram nível III $(14,2 \%)$ e dois participantes nível II (7,1\%). Não foram encontrados participantes com nível motor I. Os participantes não foram avaliados a nível de independência funcional, linguagem ou cognição.

Todos foram avaliados pela escala de SAROMM e os resultados obtidos foram comparados com dados do próprio sujeito, como idade e nível motor (GMFCS). A análise da Tabela 1 permite verificar que os participantes que apresentam maior pontuação na escala SAROMM apresentam nível motor V e IV (78,5\%). 
Tabela 1. Resultados gerais da avaliação dos participantes através da Spinal Alignment and Range of Motion Measure (SAROMM)

\begin{tabular}{|c|c|c|c|c|c|c|c|c|}
\hline Participantes & $\begin{array}{c}\text { Alinhamento } \\
\text { Espinhal }\end{array}$ & $\begin{array}{l}\text { Escore } \\
\text { quadril }\end{array}$ & $\begin{array}{l}\text { Escore } \\
\text { joelho }\end{array}$ & $\begin{array}{l}\text { Escore } \\
\text { tornozelo }\end{array}$ & $\begin{array}{l}\text { Escore } \\
\text { MMSS }\end{array}$ & ADM & $\begin{array}{c}\text { Total } \\
\text { SAROMM }\end{array}$ & Idade \\
\hline Participante 1 & 3,2 & 2 & 2 & 3 & 1 & 2 & 59 & 6 \\
\hline Participante 2 & 3 & 2 & 2 & 2 & 2 & 2 & 56 & 32 \\
\hline Participante 3 & 2 & 2,3 & 2 & 3 & 2 & 2,3 & 60 & 37 \\
\hline Participante 4 & 2 & 2,4 & 2,5 & 1 & 2 & 1,9 & 55 & 35 \\
\hline Participante 5 & 2 & 1,5 & 2,5 & 0,2 & 1 & 1,3 & 38 & 25 \\
\hline Participante 6 & 1,2 & 0,5 & 1 & 1 & 0 & 0,6 & 19 & 20 \\
\hline Participante 7 & 4 & 3,1 & 4 & 4 & 3 & 3,5 & 92 & 21 \\
\hline Participante 8 & 1,7 & 2,7 & 4 & 3 & 3 & 3,1 & 74 & 21 \\
\hline Participante 9 & 3,7 & 3,2 & 4 & 4 & 4 & 3,8 & 94 & 28 \\
\hline Participante 10 & 2,4 & 3,3 & 3 & 2 & 1 & 2,3 & 72 & 23 \\
\hline Participante 11 & 2 & 2,3 & 2 & 2 & 1 & 1,8 & 54 & 25 \\
\hline Participante 12 & 3 & 3 & 3,7 & 3 & 3 & 3,1 & 81 & 32 \\
\hline Participante 13 & 2 & 2,2 & 2,5 & 2 & 1 & 1,9 & 54 & 19 \\
\hline Participante 14 & 2 & 2 & 2,5 & 2 & 1 & 1,8 & 48 & 21 \\
\hline Participante 15 & 4 & 2,1 & 3 & 3 & 1 & 2,2 & 60 & 13 \\
\hline Participante 16 & 2,5 & 1,3 & 2 & 1 & 0 & 1,0 & 35 & 14 \\
\hline Participante 17 & 2,7 & 3,1 & 3 & 3 & 2 & 2,7 & 77 & 36 \\
\hline Participante 18 & 2 & 2 & 2,5 & 0,7 & 1 & 1,5 & 45 & 15 \\
\hline Participante 19 & 2,5 & 2,3 & 2 & 2 & 2 & 2,0 & 58 & 21 \\
\hline Participante 20 & 3,7 & 3,2 & 4 & 4 & 2 & 3,3 & 90 & 27 \\
\hline Participante 21 & 1,7 & 2,1 & 4 & 2 & 3 & 2,7 & 63 & 21 \\
\hline Participante 22 & 3,7 & 3 & 3 & 2 & 2 & 2,5 & 77 & 21 \\
\hline Participante 23 & 3 & 3,3 & 4 & 4 & 3,5 & 3,7 & 91 & 23 \\
\hline Participante 24 & 3 & 3,5 & 3 & 2 & 4 & 3,1 & 82 & 25 \\
\hline Participante 25 & 3,7 & 2,9 & 2,5 & 3,5 & 3 & 2,9 & 80 & 15 \\
\hline Participante 26 & 2 & 2,0 & 2,5 & 1 & 3 & 2,1 & 53 & 8 \\
\hline Participante 27 & 2 & 2 & 3 & 2 & 2 & 2,2 & 56 & 11 \\
\hline Participante 28 & 3,2 & 3,1 & 4 & 4 & 4 & 3,7 & 91 & 15 \\
\hline Valor médio & 2,6 & 2,4 & 2,8 & 2,3 & 2,0 & 2,4 & 64,7 & 21,7 \\
\hline
\end{tabular}

MMSS: membro superior; ADM: amplitude de movimento.

GMFCS V
GMFCS IV
GMFCS III
GMFCS II


As articulações mais acometidas por deformidades e encurtamentos musculares foram joelhos, coluna vertebral e quadril respectivamente (Figura 1). Sujeitos maiores de 20 anos, obtiveram uma pontuação média de 68,7. Considerando que 52 seria a pontuação mediana da escala SAROMM (104 é o máximo), ou seja, sujeitos com mais deformidades e encurtamentos, os sujeitos mais velhos obtiveram mais deformidades e encurtamentos que os mais jovens, que também obtiveram uma pontuação próximo de $52 \%(55,1)$. O sujeito com maior pontuação tinha 28 anos (SAROMM 94) e o de menor pontuação tinha 20 anos (SAROMM 19). O mais jovem pontuou 59 pontos (6 anos) e o mais velho 60 pontos (37 anos).
Neste estudo, houve uma grande proporção de indivíduos com contraturas e deformidades já instaladas, sendo mais constante na articulação dos joelhos, coluna e quadril respectivamente, lembrando que a escala SAROMM utiliza-se da goniomentria. $\mathrm{Na}$ avaliação topográfica (Figura 2) observou que o predomínio do grupo avaliado constava de pacientes com PC do tipo espástico bilateral (quadriplegia) com 19 participantes $(67,8 \%)$, seguido de espástico bilateral com predomínio de membros inferiores com seis participantes $(21,4 \%)$ e, por fim, dois participantes com espástico unilateral (7\%). $\mathrm{O}$ quadro de espasticidade predominou em todo o grupo avaliado $(96,4 \%)$.

Articulações mais acometidas

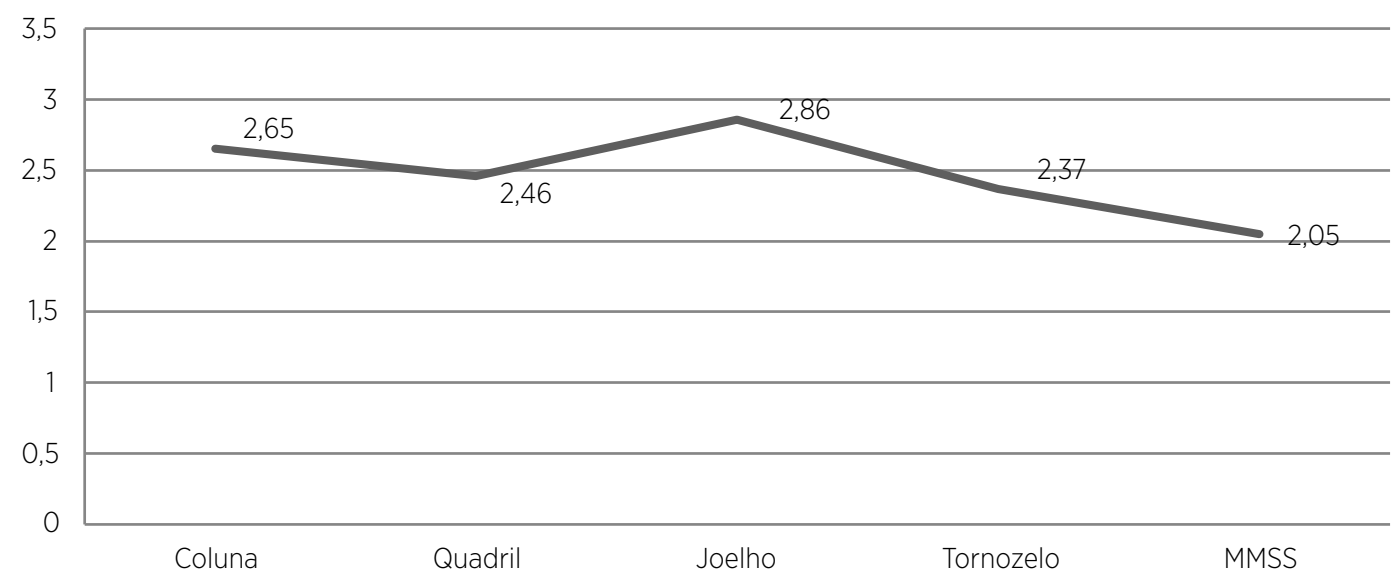

MMSS: membros superiores

Figura 1. Articulações mais acometidas por encurtamentos e deformidades

Distribuição topográfica

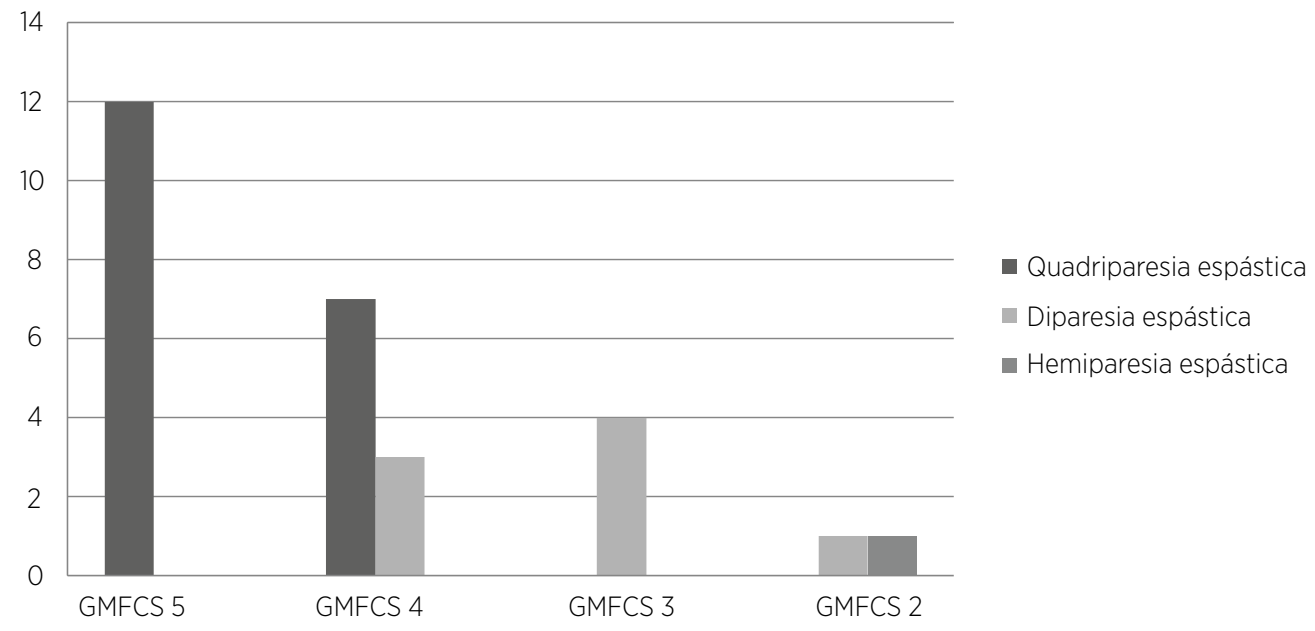

GMFCS: Gross Motor Function Classification System.

Figura 2. Acometimento topográfico dos indivíduos avaliados 
O curso natural da PC mudou bastante durante os últimos 50 anos. Estudos em vários países têm demonstrado que a expectativa de vida de indivíduos com PC tem aumentado ${ }^{18}$. As crianças com PC podem ter sobrevivência semelhante à da população em geral, quando não apresentam comorbidades significativas e recebem cuidados de saúde adequados ${ }^{19}$.

Felizmente, nos últimos dez anos, as equipes de saúde vêm detectando precocemente as lesões neurofuncionais das crianças e iniciando um tratamento de reabilitação o mais cedo possível. A avaliação inicial do cérebro após a lesão é desafiadora, pois há uma incerteza para saber em que momento ocorreu o evento da lesão neurológica. Os exames complementares (eletroencefalograma, ecografía cerebral, líquido cefalorraquidiano, ressonância magnética) e a clínica do paciente são grandes aliados para o diagnóstico precoce ${ }^{20,21}$. No entanto, sujeitos que nasceram há mais tempo, não obtiveram o mesmo desfecho. No caso de moradores de abrigo mais antigos, o diagnóstico da PC era feito tardiamente, não se investia em um tratamento, principalmente porque era considerado longo e que gerava dor ao mesmo. O seguimento era desaconselhado ou pouco compreendido. $\mathrm{O}$ sujeito morador de abrigo não tinha uma família estruturada que pudesse investir no seu desenvolvimento ${ }^{22}$.

O que se pode fazer com essa população, onde as sequelas já estão instaladas? O importante é tentar diminuir ou minimizá-las. É de extrema importância conhecer o perfil dos sujeitos, o local onde moram e as alterações musculoesqueléticas, como o alinhamento da coluna vertebral e a ADM. Outro objetivo é traçar estratégias para minimizar o avanço das deformidades já instaladas.

Assim como no presente estudo, Andersson e Mattsson ${ }^{23}$ encontraram $80 \%$ de contraturas em adultos com PC. Dos 27 participantes quadriplégicos, 25 não deambulavam e, dentre estes, apenas um não possuía contratura. Dos 47 hemiplégicos, 31 possuíam contraturas. De acordo com os resultados de estudos de ultrassonografia em músculos de sujeitos com $\mathrm{PC}$, tanto em casos da imobilidade ou desuso como em casos de uso em excesso ou em situação biomecânica desfavorável, ocorrem adaptações teciduais e, como consequências, ocorrem contraturas, atrofias musculares e modificações na arquitetura muscular. O músculo é um órgão dinâmico que se adequa às demandas de sua utilização.

Além do comprometimento bilateral dos membros, a espasticidade também predomina na $\mathrm{PC}$. Os pacientes espásticos apresentam alteração global de tônus muscular, diminuição da motricidade espontânea e da mobilidade articular, propiciando o surgimento de deformidades. Já os espásticos bilaterais com predomínio de membros inferiores e os espásticos unilaterais, em geral, apresentam menor alteração de tônus muscular em relação aos espásticos bilaterais dos quatro membros ${ }^{24}$. Normalmente, essas alterações biomecânicas levam à assimetria, alterações de descarga de peso, alterações do equilíbrio, ou seja, utilizam-se de estratégias compensatórias para realizar suas funções ${ }^{8}$. Como neste estudo, a espasticidade dos quatro membros foi predominante $(67,8 \%)$, entende-se que a funcionalidade é bastante limitada. A maior parte do tempo em camas e cadeiras. Esse cérebro está muito imaturo e mais suscetível a essas lesões. Já para a espasticidade unilateral, Santos sugere que a prevalência seja de $21-40 \%$ dos casos de PC. Neste estudo, encontramos $7 \%{ }^{15}$.

Para este público, entende-se que, quanto mais grave é o caso da criança, mais risco de abandono ocorre pela família biológica, então, mais pacientes com lesão espástica e bilateral dos quatro membros ${ }^{25}$. Nas fases mais precoces, a espasticidade leva a posicionamentos articulares inadequados, acarretando contraturas musculares dinâmicas que, com o passar do tempo, tornam-se fixas, levando a deformidades articulares. Ocorre um desequilibrio muscular entre agonistas e antagonistas, e contração/tensão excessiva dos músculos espásticos que distende e enfraquece seus antagonistas. É importante destacar que existem deformidades secundárias em virtude das compensações, como, por exemplo, a flexão do quadril ser secundário à flexão de joelho, e o equino ser secundário à flexão de joelho. Isso se deve ao fato de muitos músculos terem ação em mais de uma articulação, fato recorrente na nossa amostra ${ }^{25}$.

As alterações na coluna vertebral foram uma das mais encontradas. A escoliose nas crianças com PC é decorrente de posturas inadequadas, que podem afetar negativamente na morfologia do tórax e fisiologia do sistema respiratório, acarretando a ocorrência de comorbidades respiratórias que afetarão a qualidade de vida destas crianças ${ }^{26}$. Foram encontrados índices elevados de escoliose no grupo dos espástico bilaterais dos quatro membros. Entre as crianças com essa alteração na coluna, nenhuma apresentava marcha. Hodgkinson et al. ${ }^{27}$ relataram que as crianças com PC que não deambulam apresentaram porcentagem elevada de ocorrências de postura escoliótica. As deformidades relacionadas ao tronco de pacientes espásticos são observadas escolioses graves, com maior incidência em região toracolombar.

Bottos et al. ${ }^{28}$ em um estudo de caracterização de 72 adultos com PC na Itália, encontraram escoliose acima de $30^{\circ}$ em $20,3 \%$ dos participantes e $28,2 \%$ de luxação 
ou subluxação de quadris. Turk et al. ${ }^{29}$ em um estudo com 63 mulheres adultas com PC, encontraram 40\% de deformidades de quadril (obliquidade pélvica ou luxação de quadril), 53\% de deformidades na coluna (cifose ou escoliose) e $75 \%$ de contraturas musculares. No presente estudo 14 participantes $(50 \%)$ apresentaram maiores deformidades na articulação do joelho, 10 participantes na coluna vertebral $(35,7 \%)$, e quatro participantes com deformidades em quadril $(14,2 \%)$. As deformidades no quadril foram distribuídas em rotação interna, rotação externa e flexão, sendo esta última mais evidente no grupo dos espásticos bilaterais de membros inferiores. As rotações internas e externas do quadril acontecem em virtude da anormalidade dos músculos psoas, rotadores internos e externos, adutores e pela anteversão exagerada do colo femoral, geralmente encontrada nas crianças com PC que não deambulam.

A obliquidade pélvica está associada às curvas neuropáticas que impedem assim que a pelve mantenha sua horizontalidade na posição sentada. Decorrente dessa alteração, a rotação pélvica pode se desenvolver por causa de contraturas dos músculos que se fixam acima e abaixo da pelve. São comuns encontrarmos a luxação coxofemoral, levando a uma limitação da abdução passiva e ativa do quadril. Por fim, a deformidade em varismo dos pés é consequência da espasticidade do músculo tibial posterior. Porém, em nossa pesquisa foi encontrado o valgismo dos pés associado a espasticidade dos fibulares e o equinismo do pé, seguido de hálux valgo e artelhos dos pés em garra ${ }^{30}$. Em membros superiores, as alterações ortopédicas que mais se destacaram neste estudo foram: rotação interna de ombro, flexão de cotovelo e punho, desvio ulnar e oponência do polegar. $\mathrm{O}$ uso de órtese pode ser uma excelente estratégia para este tipo de população ${ }^{31}$.

Neste estudo, sujeitos maiores de 20 anos, obtiveram uma pontuação média de SAROMM maior que os menores de 20 anos. $\mathrm{O}$ aumento da idade entre indivíduos com nível GMFCS IV e $V$ foi associada com as pontuações mais elevadas da SAROMM. No entanto, devemos sempre pensar que a gravidade, a progressão, o aumento dos encurtamentos musculares e aparecimento de deformidades é uma característica de cada sujeito e de sua história. Depende do tamanho, do volume da lesão, quando ela ocorre e como ela e suas sequelas são interpeladas ${ }^{32}$. Os danos provocados no sistema nervoso imaturo associado à assistência terapêutica tardia, muitas vezes decorrente da falta de conhecimento dos pais sobre reabilitação e estimulação, acarretarão o aparecimento de encurtamentos musculares, levando a um prognóstico insatisfatório relacionado ao desenvolvimento neuropsicomotor de crianças com PC.
Uma estratégia para minimizar a evolução das deformidades e encurtamentos de indivíduos com PC com quadro motor mais grave (GMFCS V e IV), de uma instituição de abrigagem é realizar um programa de orientação aos cuidadores destes sujeitos, bem como ensinar posicionamentos adequados na cadeira de rodas e leito, alongamentos passivos das articulações, a adesão a um serviço de reabilitação e o uso de dispositivos ortopédicos como órteses de MMII e MMSS.

Este estudo tem alguns pontos fortes notáveis. Primeiro, realizou-se este estudo em um local com atendimento para abrigados com PC de excelência na nossa cidade. Discutiu-se que, o que foi perdido em relação às questões funcionais dos abrigados mais velhos deve ser detectado e corrigido para os próximos abrigados que ingressarem na Instituição. Esta geração futura de abrigados pode iniciar a fisioterapia mais precocemente, com uso de órteses e com melhores posturas. Também, sempre os cuidadores devem ser orientados para saber como auxiliar na reabilitação e nas atividades de vida diária. Com o aumento do conhecimento, os cuidadores podem solicitar a equipe de reabilitação exames complementares, melhores diagnósticos e prognósticos. O tratamento fisioterapêutico na $\mathrm{PC}$ espástica poderá atuar, não só no ganho de amplitude articular e força, mas também, facilitar o movimento para as atividades de vida diária (alimentação, locomoção, transferência e higienização).

As limitações deste estudo foram que: as análises estatísticas poderiam ter sido melhores exploradas havendo correlações; também se encontrou dados incompletos para análise da história pregressa nos prontuários dos pacientes, o que limitou na exploração da história inicial da vida dos sujeitos, podendo correlacionar com as características das mesmas. Seria interessante considerar um convite a outras Instituições de abrigagem com esse tema como verificar os dados de seus sujeitos com PC e compará-los com outros locais.

\section{CONCLUSÃO}

Este estudo mostrou que os sujeitos com PC de um local de abrigagem apresentaram, na sua maioria, espasticidade do tipo bilateral dos quatro membros, nível de GMFCS predominante foi o $\mathrm{V}$ e alterações articulares em joelhos, quadril e coluna vertebral como maiores deformidades encontradas. As deformidades e encurtamentos aumentam com o tempo, mas não são idades dependentes, e sim, do tamanho da lesão, do volume e da história de cada um. 
As estratégias que podem ser traçadas são de iniciar o mais cedo possível as trocas de posturas (sedestação e ortostase) para aqueles sem deformidades instaladas. Para os PC com alterações musculoesqueléticas, buscar um alinhamento da coluna vertebral e estratégias para minimizar o avanço das deformidades já instaladas. Envolver toda a equipe nestes procedimentos seria uma estratégia bastante relevante para a melhoria dos resultados, principalmente para os próximos ingressantes no abrigo.

Sugere-se que o fisioterapeuta possa contar com uma nova forma de avaliação pré e pós intervenção terapêutica, e a escala SAROMM pode ser uma escolha acertada.

\section{REFERÊNCIAS}

1. Lier-Devitto MF, Dudas TL. Institucionalização de pessoas com paralisia cerebral: a difícil relação sujeito-outro-linguagem. Lingüística. 2016;32(1):9-23. doi: 10.5935/2079-312X.20160001

2. Wotherspoon J, Whittingham K, Boyd RN, Sheffield J. Randomised controlled trial of a novel online cognitive rehabilitation programme for children with cerebral palsy: a study protocol. BMJ Open. 2019;9(6):e028505. doi: 10.1136/bmjopen-2018-028505

3. The American College of Obstetricians and Gynecologists. ACOG Committee Opinion, number 326: inappropriate use of the terms fetal distress and birth asphyxia. Obstet Gynecol. 2005;106(6):1469-70. doi: 10.1097/00006250-200512000-00056

4. Pfeifer LI, Silva DBR, Funayama CAR, Santos JL. Classification of cerebral palsy: association between gender, age, motor type, topography and gross motor function. Arq Neuro-Psiquiatr. 2009;67(4):1057-61. doi: 10.1590/S0004-282X2009000600018

5. Centers for Disease Control and Prevention. Data and statistics for cerebral palsy [Internet]. Atlanta: Centers for Disease Control and Prevention; [cited 2020 Nov 5]. Available from: https://www.cdc.gov/ncbddd/cp/data.html

6. Novak I, Morgan C, Adde L, Blackman J, Boyd RN, BrunstromHernandez J, et al. Early, accurate diagnosis and early intervention in cerebral palsy: Advances in diagnosis and treatment. JAMA Pediatr. 2017;171(9):897-907. doi: 10.1001/jamapediatrics.2017.1689

7. Catena F, Moraes ER, Lemos AVKC, Yamane PC, Blumetti FC, Dobashi ET, Pinto JA. Estudo clínico do quadril não tratado na tetraparesia espástica. Rev Bras Ortop. 2011;46 (Suppl 4);21-6. doi: 10.1590/S0102-36162011001000005

8. Brasil. Ministério da Saúde. Diretrizes de atenção à pessoa com paralisia cerebral. Brasília, DF: Ministério da Saúde; 2013.

9. Olama KA, Kassem HI, Aboelazm SN. Impact of aquatic exercise program on muscle tone in spastic hemiplegic children with cerebral palsy. Clin Med J. 2015;1(4):138-44

10. Silva EM, Silva TAS, Balk RS, Lopes RR, Santos CC, Lara S, et al. Avaliação do alinhamento postural e extensibilidade muscular pela escala SAROMM em crianças com paralisia cerebral após fisioterapia aquática. Fisioter Bras. 2017;18(6):719-26. doi: 10.33233/fb.v18i6.2054
11. Agut T, Póo P, Launes C, Auffant M, Iriondo M. Incidence of cerebral palsy in a cohort of preterm infants with a gestational age of less than 28 weeks.An Pediatr (Barc). 2015;82(1):49-50. doi: 10.1016/j.anpedi.2013.12.016

12. Monteiro CBM. Realidade virtual na paralisia cerebral. São Paulo: Plêiade; 2011.

13. Dinomais M, Hertz-Pannier L, Groeschel S, Chabrier S, Delion M, Husson B, et al. Long term motor function after neonatal stroke: Lesion localization above all. Hum Brain Mapp. 2015;36(12):4793-807. doi: 10.1002/hbm.22950

14. Ministerio de Sanidad, Servicios Sociales e Igualdad. Guía de práctica clínica sobre encefalopatía hipóxico-isquémica perinatal en el recién nacido. Madrid: Ministerio de Sanidad, Servicios Sociales e Igualdad; 2015.

15. Santos AF. Paralisia cerebral: uma revisão da literatura. Unimontes Cient. 2015;16(2):67-82.

16. Bartlett D, Purdie B. Testing of the spinal alignment and range of motion measure: a discriminative measure of posture and flexibility for children with cerebral palsy. Dev Med Child Neurol. 2005;47(11):739-43. doi: 10.1017/S0012162205001556

17. CanChild Centre for Childhood Disability Research. GMFCS E \& R: Sistema de Classificação da Função Motora Grossa ampliado e revisto. Hamilton: CanChild; 2007.

18. Hemming K, Hutton JL, Pharoah PO. Long-term suvival for a cohort of adults with cerebral palsy. Dev Med Child Neurol. 2006;48(2):90-5. doi: 10.1017/S0012162206000211

19. Donkervoort M, Roebroeck M, Wiegerink D, van der HeijdenMaessen H, Stam H, Transition Research Group South West Netherlands. Determinants of functioning of adolescents and young adults with cerebral palsy. Disabil Rehabil. 2007;29(6):453-63. doi: 10.1080/09638280600836018

20. Filho PCN, Duarte FT, Fortes JPA, Júnior FFUS. Alterações no comportamento elétrico cerebral de uma criança com paralisia cerebral após atendimento com neurofeedback. Fisioter Bras. 2017;18(3):369-73. doi: 10.33233/fb.v18i3.1065

21. Echeverría-Palacio CM, Agut T, Arnaez J, Valls A, Reyne M, GarciaAlix A. Neuron-specific enolase in cerebrospinal fluid predicts brain injury after sudden unexpected postnatal collapse. Pediatr Neurol. 2019;101:71-7. doi: 10.1016/j.pediatrneurol.2019.02.020

22. Ribeiro MFM, Vandenberghe L, Prudente COM, Vila VSC, Porto CC. Cerebral palsy: how the child's age and severity of impairment affect the mother's stress and coping strategies. Ciênc Saúde Coletiva. 2016;21(10):3203-12. doi: 10.1590/1413-812320152110.17352016

23. Andersson C, Mattsson E. Adults with cerebral palsy: a survey describing problems, needs, and resources, with special emphasis on locomotion. Dev Med Child Neurol. 2001;43(2):76-82. doi: 10.1111/j.1469-8749.2001.tb00719.x

24. Ávila ASC, Rocha CAQ. Atuação fisioterapêutica em paciente com PC com tetraparesia espástica assimétrica: um estudo de caso. Rev Cient Faminas. 2014;10(2):21-7.

25. Gomes CO, Golin MO. Tratamento fisioterapêutico na paralisia cerebral tetraparesia espástica, segundo conceito Bobath. Rev Neurociênc. 2013;21(2):278-85. doi: 10.4181/RNC.2013.21.757.8p

26. Fernandes MV, Fernandes AO, Franco RC, Golin MO, Santos LA, Setter CM, et al. Adequações posturais em cadeira de rodas: prevenção de deformidades na paralisia cerebral. Rev Neurociênc. 2007;15(4):292-6. 
27. Hodgkinson I, Bérard C, Chotel F, Bérard J. Pelvic obliquity and scoliosis in non-ambulatory patients with cerebral palsy: a descriptive study of 234 patients over 15 years of age. Rev Chir Orthop Reparatrice Appar Mot. 2002;88(4):337-41.

28. Bottos M, Feliciangeli A, Sciuto L, Gericke C, Vianello A. Functional status of adults with cerebral palsy and implications for treatment of children. Dev Med Child Neurol. 2001;43(8):516-28. doi: 10.1111/j.1469-8749.2001.tb00755.x

29. Turk MA, Geremski CA, Rosenbaum PF, Weber RJ. The health status of women with cerebral palsy. Arch Phys Med Rehabil. 1997;78(12):S10-7. doi: 10.1016/s0003-9993(97)90216-1
30. Mazzitelli C, Amaral, PP, Mazzitelli, C. Alterações ortopédicas em crianças com paralisia cerebral da clínica-escola de fisioterapia da Universidade Metodista de São Paulo (Umesp). Rev Neurociênc. 2003;11(1):29-33.

31. Ireno JM, Chen N, Zafani MD, Baleotti LR. The use of orthoses in children with cerebral palsy: perception of caregivers. Cad Bras Ter Ocup. 2019;27(1):35-44. doi: 10.4322/2526-8910.ctoao1612

32. Arca G, Arnaez J, Agut T, Núñez C, Stephan-Otto C, Valls $A$, et al. Neuron-specific enolase is correlated with lesion topology, relative infarct volume and outcome of symptomatic NAIS. Arch Dis Child Fetal Neonatal. 2019;105(2):132-37. doi: 10.1136/archdischild-2018-316680 